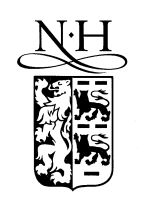

ELSEVIER

Applied Surface Science 138-139 (1999) 102-106

applied

surface science

\title{
Modeling of velocity and surface temperature of the moving interface during laser ablation of polyimide and poly(methyl methacrylate)
}

\author{
H. Schmidt ${ }^{\mathrm{a}, 1}$, J. Ihlemann ${ }^{\mathrm{a}, *}, \mathrm{~K}$. Luther ${ }^{\mathrm{b}}$, J. Troe ${ }^{\mathrm{b}}$ \\ ${ }^{a}$ Laser-Laboratorium Göttingen, Hans-Adolf-Krebs-Weg 1, D-37077 Göttingen, Germany \\ ${ }^{\mathrm{b}}$ Inst. f. Physikalische Chemie, Univ. Göttingen, Tammannstr. 6, D-37077 Göttingen, Germany
}

\begin{abstract}
A recently developed model, which is very successful in explaining pulse duration and spot size dependences of nanosecond laser ablation rates of polyimide (PI) and poly(methyl methacrylate) (PMMA) [H. Schmidt, J. Ihlemann, B. Wolff-Rottke, K. Luther, J. Troe, J. Appl. Phys., 83 (1998) 5458.], is applied to calculate the temporal behavior of position and temperature of the moving interface between the condensed phase of the polymer sample and the ablation plume, i.e., the receding sample surface, during the ablation process. The model describes the polymer as a system of chromophores with two possible electronic states. It is based on the combination of photothermal decomposition and photodissociative bond breaking in the electronically excited state. Laser-induced chemical modifications are incorporated via different absorption coefficients for the initial and for the UV-modified polymer. Dynamic attenuation of the incoming radiation by the expanding ablation plume and heat conduction are taken into account. The model predicts maximum surface temperatures during ablation of about $3000 \mathrm{~K}$ in the case of PI and about $700 \mathrm{~K}$ in the case of PMMA. Typical maximum velocities of the moving interface range from $20 \mathrm{~m} / \mathrm{s}$ (PI) to $100 \mathrm{~m} / \mathrm{s}$ (PMMA) for a pulse duration of $20 \mathrm{~ns}$ at a fluence of $4 \mathrm{~J} / \mathrm{cm}^{2}$. The simulations show that the attenuation of the laser pulse by the plume of ejected material, which reaches a factor of up to 5 shortly after the peak of the pulse, starts to decrease towards the end of the laser pulse. These calculations support the significance of three dimensional plume expansion for the increase of ablation rates with growing pulse duration and decreasing laser spot size. (c) 1999 Elsevier Science B.V. All rights reserved.
\end{abstract}

PACS: 79.20.Ds; 61.80.Ba; 61.82.Pv; 81.65.Cf

Keywords: Laser ablation; Photoablation; Moving interface

\section{Introduction}

One of the ideas essential for realistic modeling of photoablation of polymers under wide varieties of conditions is the 'Moving interface concept'. It is assumed that during the period of some $10 \mathrm{~ns}$ of UV-laser irradiation, material is continuously evaporated, so that the resulting sample surface continu-

\footnotetext{
* Corresponding author. Tel.: +49-551-503544; Fax: +49551-503599; E-mail: jihle@1lg.gwdg.de

${ }^{1}$ Present address: MicroLas Lasersystem, Robert-Bosch-Breite

10, D-37079 Göttingen, Germany.
} 
ously recedes back from zero position until it reaches the total ablation depth at the end of the pulse or a short time later [2-4]. This behavior has also been proved experimentally by time resolved photography of the surface during the ablation process [5].

In this paper, we present calculations of the time evolution of surface temperature and velocity of the moving surface during ablation, which are based on a recently developed ablation model. The significance of this model was demonstrated by its capability to consistently explain the dependence of measured ablation rates on laser spot sizes and pulse durations as well as the plume attenuation results, which were obtained in experiments with various materials and wavelengths, especially in the medium and high fluence range [1].

\section{Model}

A detailed description of the model is given in Ref. [1]. In the following sentences below we give a short summary. Absorption of laser radiation leads to excitation of chromophoric units in the polymeric chains into the excited state. Deactivation processes are considered as either direct bond breaking in the excited electronic state (i.e., 'photodissociation') or electronic relaxation, i.e., the internal conversion into a vibrationally very hot electronic ground state molecule, in which unimolecular bond breaking of the hot molecules competes with vibrational relaxation. The latter leads to heating of the surrounding material with the eventual possibility of decomposition via thermal activation. Bond breaking leads to modified chromophores with different absorption cross-sections. Ablation of a layer occurs, if the number of broken bonds exceeds a threshold value. The plume of ablated material expands laterally and continues to absorb photons where it interferes with the laser beam.

The polymer substrate is treated as a system of infinitesimally thin layers, each with the concentrations $n_{0}$ and $n_{1}$ which are given by the sum of original and modified chromophores in the $S_{0}$ state and in the $S_{1}$ state, respectively. The temporal evolution of $n_{1}$ and that of the concentration of modified chromophores $n_{\text {mod }}$ is described by a rate equation system using rate constants $k_{\mathrm{dd}}$ for direct decompo- sition and $k_{\mathrm{th}}$ for thermal decomposition according to an Arrhenius law. These reaction pathways are shown in Fig. 1.

If for a layer the ablation condition $n_{\bmod } \geq n_{\mathrm{tr}}\left(n_{\mathrm{tr}}\right.$ threshold concentration) is reached, the molecules of this layer will leave the solid polymer substrate with high velocity. The direction of this particle movement is described by a $\cos ^{\mathrm{n}}(\beta)$-distribution [6,7]. ( $\beta$ is the angle between the direction of movement and the surface normal). To simplify the calculation, we made the following assumptions (Fig. 2):

1. There is an angle $\beta$, which limits this lateral plume expansion. The lateral distribution of the particle concentration within this limiting angle remains constant.

2. The particles are moving with a velocity, which has a constant component $\nu$ in $x$-direction (parallel to the surface normal).

3. The ablating laser beam has a constant radius $r$ above the sample.

4. The absorptivity of the plume particles is no longer changed by absorption processes.

Under these conditions, the total absorption cross-section $\sigma$ of the ablated layer decreases during its expansion according to

$\sigma(t)=\sigma_{\bmod } \cdot f(t)$

with the absorption cross-section at the ablation threshold $\sigma_{\bmod }$ and

$f=\left[1+\frac{\nu t}{r} \cdot \tan (\beta)\right]^{-2}$

Each layer leads to an attenuation of the incident laser light:

$$
\frac{\mathrm{d} I}{\mathrm{~d} x}=-\sigma_{\mathrm{mod}} \cdot f \cdot n_{0} \cdot I
$$

Scattering and reflection are taken into account by a constant factor of $6 \%$ [8]. Now the temperature of

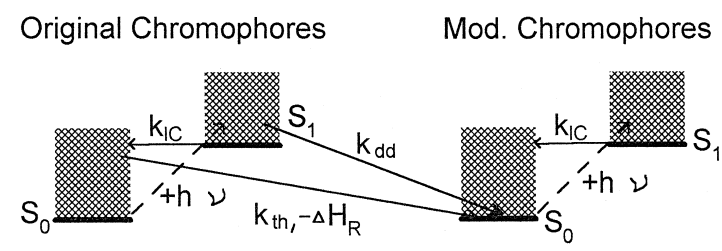

Fig. 1. Scheme of reaction pathways considered in the model. 


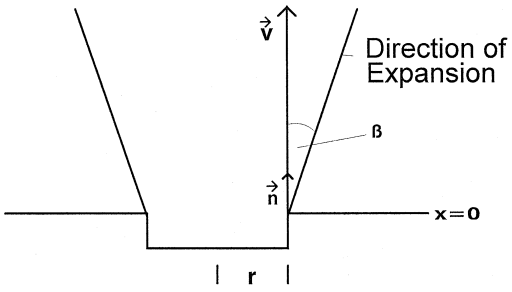

Fig. 2. Geometrical parameters describing the movement of the ejected material.

each polymer layer can be calculated. The increase of temperature due to internal conversion is given by ( $c_{\mathrm{p}}$ specific heat capacity):

$$
\left.\frac{\mathrm{d} T}{\mathrm{~d} t}\right|_{\mathrm{IC}}=-\left.\frac{\mathrm{d} n_{1}}{\mathrm{~d} t}\right|_{\mathrm{IC}} \cdot \frac{h \cdot \nu}{c_{\mathrm{p}}}
$$

Thermal decomposition out of $S_{0}$ consumes the reaction enthalpy $\Delta H_{\mathrm{R}}$ leading to a change in temperature:

$$
\left.\frac{\mathrm{d} T}{\mathrm{~d} t}\right|_{\mathrm{th}}=\left.\frac{\mathrm{d} n_{\mathrm{mod}}}{\mathrm{d} t}\right|_{\mathrm{th}} \cdot \frac{\Delta H_{\mathrm{R}}}{c_{\mathrm{p}}}
$$

For direct bond breaking the influence on the temperature is neglected, the energy difference between $S_{0}$ and $S_{1}$ is assumed to be equal to $\Delta H_{\mathrm{R}}$. A temperature change caused by heat conduction (heat conductivity $\chi$ ) to other layers along the $x$-axis is given by

$$
\left.\frac{\partial T}{\partial t}\right|_{x=\text { const. }}=\left.\frac{\chi}{c_{\mathrm{p}}} \cdot \frac{\partial^{2} T}{\partial x^{2}}\right|_{t=\text { const. }}
$$

The change of heat capacity $c_{\mathrm{p}}$ with temperature is accounted for by a linear approximation. A possible saturation at high temperatures is neglected for the purpose of simplicity. The heat conductivity was set constant according to Ref. [9], its influence on the calculated ablation rates is negligible. Heat conduction between polymer surface and the material in the plume and in lateral directions is neglected.

The calculation proceeds as follows: Time (during laser pulse and some time beyond) and space (polymer sample in the $x$-direction) are subdivided into a fixed number of slices. Starting with ambient temperature, $n_{1}=0$, and $n_{\text {mod }}=0$, for every time and every position (depth) $x$, the temperature, the concentrations $n_{0}, n_{1}$, the fractions of modified chro-
Table 1

Parameters for best fits of pulse duration and spot size dependence of the ablation rate

\begin{tabular}{lll}
\hline Parameter & PI $(248 \mathrm{~nm}, 308 \mathrm{~nm})$ & PMMA $(248 \mathrm{~nm})$ \\
\hline $\begin{array}{l}\text { Limiting angle of } \\
\text { plume expansion }\end{array}$ & $0.35 \mathrm{rad}$ & $0.25 \mathrm{rad}$ \\
$\begin{array}{l}\text { Velocity of ablated } \\
\text { material }\end{array}$ & $9 \times 10^{3} \mathrm{~m} / \mathrm{s}$ & $4 \times 10^{3} \mathrm{~m} / \mathrm{s}$ \\
$\begin{array}{l}\text { Broken bonds per } \\
\text { chromophore at the } \\
\text { ablation threshold }\end{array}$ & 0.1 & 0.07 \\
\hline
\end{tabular}

mophores in these concentrations, and the intensity $I$ are calculated. If the ablation condition is reached in a layer, this layer is considered as a plume layer. For plume layers only the absorption with a constant fraction of modified chromophores, but with expanding lateral dimensions is effective for the calculation, temperature effects are neglected. For the next pulse, the ablated depth and the values of $n_{\text {mod }}$ for the remaining layers define the initial conditions, the temperature is set to the ambient temperature and $n_{1}=0$.

\section{Results and discussion}

Using known or estimated values for the absorption coefficient of original and UV-modified polymer, and the rate constants for direct bond breaking and thermal decomposition, the best fits for the experimental results of the ablation rates under varia-

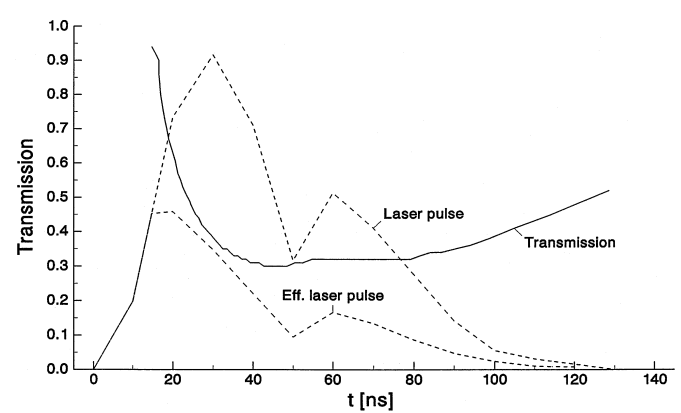

Fig. 3. Pulse shape of the original laser pulse, the laser pulse actually hitting the sample surface, and the time resolved transmission of the plume. Simulation for PI at $308 \mathrm{~nm}$, irradiation spot diameter: $116 \mu \mathrm{m}$, fluence: $3.75 \mathrm{~J} / \mathrm{cm}^{2}$, 10th pulse, starting with hole depth $30 \mu \mathrm{m}$. 


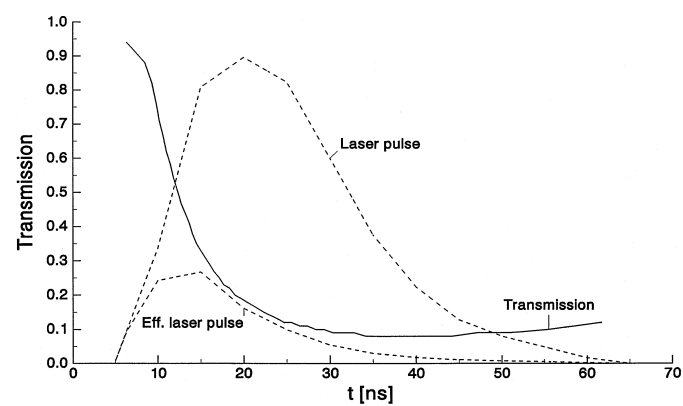

Fig. 4. Pulse shape of the original laser pulse, the laser pulse actually hitting the sample surface, and the time resolved transmission of the plume. Simulation for PMMA at $248 \mathrm{~nm}$, irradiation spot diameter: $216 \mu \mathrm{m}$, fluence: $3.5 \mathrm{~J} / \mathrm{cm}^{2}, 12$ th pulse, starting with hole depth $0 \mu \mathrm{m}$.

tion of pulse duration and spot size are obtained with the parameters given in Table 1 . The material properties used for the calculations are listed in Ref. [1].

The time resolved transmission of the plume and the resulting effective laser pulse hitting the sample surface calculated on the basis of these parameters is shown in Figs. 3 and 4. The simulations shown are for the 10th and the 12th pulse at the same sample site, respectively. For PMMA, the behavior is rather stable then after the incubation phase during the first pulses [10]. In the case of PI there is an ablation hole already after some 10 pulses, so that the lateral expansion of the plume starts later and later for every following pulse. The data agree very well with the measured plume attenuation [1]: Only during the first nanoseconds the laser energy is effective without losses. Then the effective part drops to some

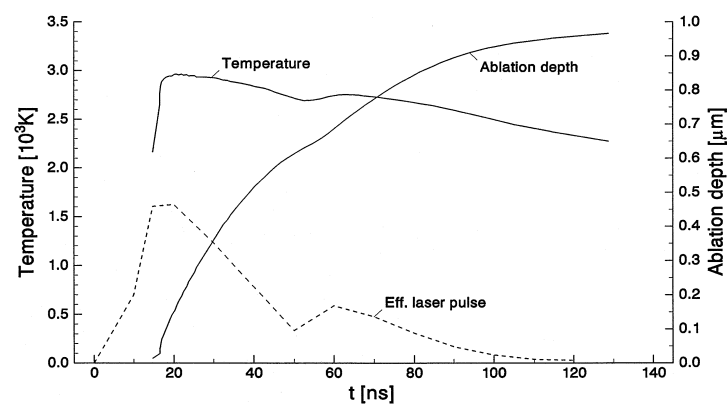

Fig. 5. Surface temperature and additional hole depth during ablation. Simulation for PI at $308 \mathrm{~nm}$, irradiation spot diameter: $116 \mu \mathrm{m}$, fluence: $3.75 \mathrm{~J} / \mathrm{cm}^{2}$, 10th pulse, starting with hole depth $30 \mu \mathrm{m}$.

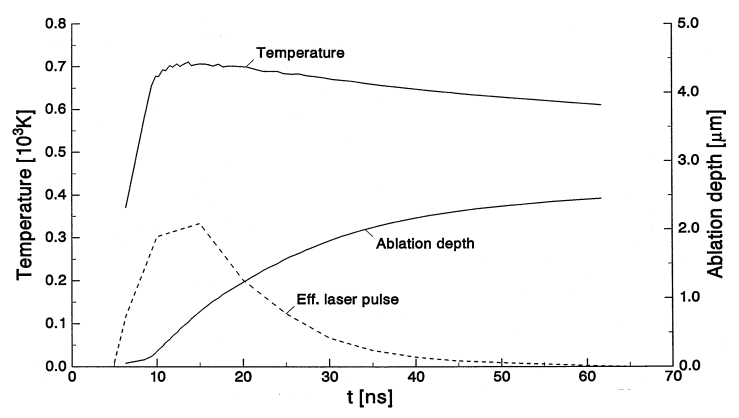

Fig. 6. Surface temperature and additional hole depth during ablation. Simulation for PMMA at $248 \mathrm{~nm}$, irradiation spot diameter: $216 \mu \mathrm{m}$, fluence: $3.5 \mathrm{~J} / \mathrm{cm}^{2}, 12$ th pulse, starting with hole depth $0 \mu \mathrm{m}$.

$10 \%$ depending on fluence, wavelength and material. At the end of the pulse, the transmission recovers due to the thinning of the plume by lateral expansion.

Figs. 5 and 6 show the time evolution of surface temperature and actual surface position during the ablation process. The laser pulse is the same as in Figs. 3 and 4, respectively. Only the effective pulse reaching the surface is shown here. After an initial rapid increase during the first part of the pulse the temperature is rather stable or slightly decreasing during the time where massive material ablation takes place. Apparently, the excess energy is carried away in the plume material. The maximum temperatures of the PI-surface are within $2000 \mathrm{~K}$ and $3600 \mathrm{~K}$ depending on pulse duration, fluence, and wavelength. The highest values were reached for short

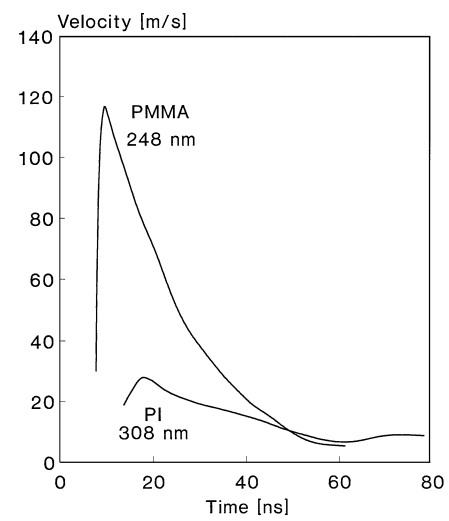

Fig. 7. Velocity of the moving interface. Data calculated for PI at $308 \mathrm{~nm}, 3.75 \mathrm{~J} / \mathrm{cm}^{2}$ and for PMMA at $248 \mathrm{~nm}, 3.5 \mathrm{~J} / \mathrm{cm}^{2}$. 
pulses at high fluence and at $248 \mathrm{~nm}$. These calculated values are in good agreement with the experimental value of $1660 \mathrm{~K}$ at the ablation threshold [9]. For PMMA the calculated maximum surface temperatures at $248 \mathrm{~nm}$ range from $450 \mathrm{~K}$ to $750 \mathrm{~K}$. Again the highest values are reached for the shortest pulse duration and highest fluence. They are comparable to the values measured for doped PMMA [11].

The calculated surface position leads directly to the velocity of the moving interface shown in Fig. 7 . Shortly after the start of ablation, the velocity reaches its maximum of about $20 \mathrm{~m} / \mathrm{s}$ for PI $(100 \mathrm{~m} / \mathrm{s}$ for PMMA) in the case of a $4 \mathrm{~J} / \mathrm{cm}^{2}$ pulse. Then, the moving interface slows down to a magnitude of $1 \mathrm{~m} / \mathrm{s}=1 \mathrm{~nm} / \mathrm{ns}$ during the trailing part of the laser pulse. Though a rough estimation of this velocity is possible by taking etch depth over pulse duration, we think that this simulation of the evolution in time gives some more understanding of how the dynamic process of polymer ablation may proceed.

\section{Conclusion}

An ablation model, which was developed on the basis of measurements of the pulse duration and spot size dependence of the ablation rate of PI and PMMA was used to calculate the surface temperature during the ablation process. These values, as well as the simulation of the plume attenuation, are in good agreement with measurements. The time resolved velocity of the moving surface was calculated.

\section{References}

[1] H. Schmidt, J. Ihlemann, B. Wolff-Rottke, K. Luther, J. Troe, J. Appl. Phys. 83 (1998) 5458.

[2] T. Keyes, R.H. Clarke, J.M. Isner, J. Phys. Chem. 89 (1985) 4194.

[3] E. Sutcliffe, R. Srinivasan, J. Appl. Phys. 60 (1986) 3315.

[4] N. Tokarev, J.G. Lunney, W. Marine, M. Sentis, J. Appl. Phys. 78 (1995) 1241.

[5] P. Simon, Appl. Phys. B 48 (1989) 253.

[6] R. Srinivasan, B. Braren, R.W. Dreyfus, J. Appl. Phys. 61 (1987) 372.

[7] S. Lazare, V. Granier, Chem. Phys. Lett. 168 (1990) 593.

[8] S. Küper: Dissertation, Göttingen, 1989.

[9] D.P. Brunco, M.O. Thompson, C.E. Otis, P.M. Goodwin, J. Appl. Phys. 72 (1992) 4344

[10] S. Küper, M. Stuke, Appl. Phys. A 49 (1989) 211.

[11] I.-Y.S. Lee, X. Wen, W.A. Tolbert, D.D. Dlott, M. Doxtader, D.R. Arnold, J. Appl. Phys. 72 (1992) 2440. 